\title{
Ich heisse Materie - und bin jemand
}

\author{
Jann Schwarzenbach \\ Dr. med., Facharzt für Allgemeinmedizin, Mitglied FMH
}

Zur alltäglichen Kontaktaufnahme mit den Dingen um uns herum bevorzugen wir in aller Regel unsere visuellen Kapazitäten. Dabei schlüpfen wir in die Rolle des distanzierten Beobachters und vernachlässigen damit die, dem Tastsinn zugänglichen, stofflich-materiellen Qualitäten. Auch bei zwischenmenschlichen Begegnungen setzen wir üblicherweise auf Abstand. Wir kommunizieren mit Hilfe von Gesten und Lauten und vermeiden dabei - Begrüssungsrituale einmal ausgenommen - tunlichst jede körperliche Berührung. Eine Ausnahme macht in dieser Hinsicht die Technik der medizinischen Palpation, wo gerade das Ertasten einer veränderten Stofflichkeit wichtige diagnostische Hinweise liefern kann. Heutzutage trägt jedoch die körperliche Untersuchung nur noch einen kleinen Teil zur Gesamtbeurteilung krankhafter Zustände bei. Sie ist zugunsten der modernen, bildgebenden Methoden

\section{In diesem Zusammenhang könnte auch die Skepsis gegenüber einer ausufernden Transplantationsmedizin verstanden werden.}

und der verschiedenartigen funktionellen und chemischen Analysen weitgehend in den Hintergrund getreten. Der Arzt und die Ärztin begegnen somit der materiellen Natur ihrer Patienten meist nur noch indirekt: im verengten Gesichtsfeld einer dicken, medizintechnischen Brille. Die stofflichen Eigenschaften des menschlichen Körpers verstecken sich dabei sozusagen hinter dem morphologisch Sichtbaren und funktionell Errechenbaren. Unter diesem Verlust an direktem Kontakt zur behandelnden Person kann dann schliesslich auch die Dignität unserer Organe leiden: Sie laufen Gefahr, zum anonymen Spielball des medizinischen Forscherdrangs zu werden.

\section{Jedes Ding an seinem Ort ...}

Solange es dabei um pharmakologische Unterstützung oder um "Reparaturarbeiten» geht, wirft das keine besonderen Probleme auf und mag für viele Patienten sogar sehr hilfreich sein. Das kann sich jedoch ändern, wenn ein Organ - ein Etwas von mir - aus seinem natürlichen Umfeld gerissen wird, um es gegebenenfalls anderenorts wieder einzupflanzen. In wenig sensiblen Bereichen wird das zwar keine grosse Rolle spielen: Aber was für die Kornea gilt, muss nicht unbedingt auch für Herz oder Lunge zutreffen. In diesem Zusammenhang könnte denn auch die weitverbreitete Skepsis gegenüber einer ausufernden Transplantationsmedizin verstanden werden. Ich möchte mich damit aber keinesfalls in die Reihe der Transplantationsgegner stellen. Es geht mir hingegen darum, die stofflichmateriellen Eigenschaften, die in der modernen Medizin allzu oft gering geschätzt werden, ein wenig zu rehabilitieren. Dies soll natürlich nicht im Rahmen eines persönlichen Plädoyers geschehen, sondern unter Zuhilfenahme der Stoa, eines philosophischen Systemdenkens, das sich über viele Jahrhunderte hinweg (ca. 300 v.Chr. bis 200 n.Chr.) erhalten und bewährt hat.

\section{Materie und ihr Logos spermatikos}

Trotz ihrer griechischen Wurzeln ist die Stoa vor allem durch ihre römischen Protagonisten wie Seneca, Epiktet und Mark Aurel populär geworden. Ihr Hauptanliegen besteht darin, den Menschen zu einem guten Leben anzuhalten, und so zeugt denn auch heute noch mancher Kalenderspruch von der zeitlosen Weisheit der obgenannten Denker. Kernpunkt der sprichwörtlichen stoischen Lebensweise ist es, nicht an den -

\section{Das heisst, dass der Lauf der Dinge den inneren} Gesetzmässigkeiten der zugrunde liegenden Materie folgen müsse.

eh unveränderbaren - Tatsachen zu verzweifeln, sondern sich umso mehr um seine inneren Werte zu kümmern. Äussere Begebenheiten sollen uns so nicht wirklich etwas anhaben können, denn persönliches Unglück beruhe vorab auf falschen Einstellungen und Fehlurteilen. Diesen lebenspraktischen Aufruf zu weitgehendem Fatalismus bei gleichzeitiger Wahrung persönlicher Tugenden, wie Pflichterfüllung an der Ge- 
meinschaft, Gerechtigkeit und Weisheitssuche, haben die berühmten stoischen Denker nicht einfach aus der Luft gegriffen. Es handelt sich dabei um eine logische Konsequenz aus den - allgemein weniger bekannten theoretischen Elementen ihrer Philosophie. Diese ist streng deterministisch und - das ist für unser Thema besonders wichtig - eindeutig materialistisch geprägt. Das heisst nun einerseits, dass alles zwingend so geschehen soll, wie es eben gerade geschieht, und andererseits, dass dieser vorbestimmte Lauf der Dinge den inneren Gesetzmässigkeiten der zugrunde liegenden Materie folgen müsse. Dies gelte nicht nur für Gegenstände und Lebewesen, sondern auch für die seelischen Regungen, für unsere Triebe und unsere Affekte. Im Gegensatz zu dem, was wir üblicherweise unter Materie verstehen, ist diese für die Stoiker eben nicht einfach etwas Formloses und Unbelebtes, sondern Träger einer eigenen, dynamischen Vernunftkraft, des sogenannten Logos spermatikos. In diesem Zusammenhang ist es von besonderer Bedeutung, dass dieser Lehre gemäss sowohl die geringsten Partikel wie auch die höchstentwickelten Strukturen in ein und denselben stoffinternen Logos eingebunden bleiben. Diese Einsicht hat grosse praktische Konsequenzen. Sie verleiht auch einfachen Dingen einen Wert und eine Würde, die es zu schützen gilt. Gleichzeitig macht sie die stoischen Philosophen zu Vorreitern einer humanen Gesellschaftsstruktur, im Sinne der Gleichstellung aller Bürger unabhängig von Rasse, Geschlecht und sozialem Status.

\section{Ein persönliches Geschenk}

Wie eingangs erwähnt, kann die allgemein übliche Vernachlässigung stofflicher Aspekte auch in unserem beruflichen Bereich durchaus negative Folgen nach sich ziehen: eine Entwürdigung der Organe und damit eine ungebremste Euphorie der Machbarkeit, gerade auch in der Transplantationsmedizin. Das darf und muss diskutiert werden. Vielleicht können die stoischen Philosophen hier ihren Beitrag zu einer persönlichen Reflexion leisten. Die Frage, ob ihre einzelnen Vertreter zu den Befürwortern oder zu den Skeptikern der Transplantation gehört hätten, kann natürlich niemand beantworten.

Im Vergleich zu vielen anderen Denkern haben sie sicher der Materie mehr Bedeutung zugemessen. Andererseits ruft aber gerade die stoische Ethik den Menschen auf, seine Pflichten der Gesellschaft gegenüber in höchstmöglichem Masse wahrzunehmen. Falls dies

\section{Philosophischen Systemen entnommene Elemente führen in der Praxis halt oft zu widersprüchlichen Schlussfolgerungen.}

durch äussere oder innere Umstände unmöglich werden sollte, wird dem Einzelnen sogar empfohlen, sein Leben selbständig zu beenden. In diesem Sinne stünde natürlich einer Organentnahme bei Hirntoten überhaupt nichts im Wege. Philosophischen Systemen entnommene Elemente führen in der Praxis halt oft zu widersprüchlichen Schlussfolgerungen, und so bleiben denn auch in der Diskussion über eine Würde unserer Organe viele Fragen offen. $\mathrm{Zu}$ viele, um in der Transplantation eindeutige Ratschläge zu geben oder gar Druck auf den Einzelnen oder auf seine Angehörigen auszuüben. So darf denn die Organspende auch nicht zur unreflektierten alltäglichen Routine werden. Sie muss und soll schlussendlich ein persönliches Geschenk bleiben. 